\title{
Implementación de una planta para la elaboración de puré de papa amarilla Tumbay deshidratada con agregados
}

\author{
Alejandro Carranza Melgar \\ Universidad de Lima \\ Ingeniería Industrial n. 28, 2010, ISSN 1025-9929, pp 95-109 \\ Recibido: 15 de junio del 2010 / Aprobado: 20 de julio del 2010
}

\begin{abstract}
Resumen: El Perú cuenta con una envidiable variedad de papas, pero su baja industrialización genera limitadas oportunidades de desarrollo a sus productores. El proyecto presentado tiene como objetivo desarrollar la cadena productiva de la papa amarilla Tumbay, en asociación con los productores y acopiadores. Su logro se alcanzará por medio de la creación de una demanda sostenible al satisfacer la necesidad de un producto nutritivo, de fácil preparación y buen sabor para el mercado peruano y, con un mayor plazo, para la exportación.
\end{abstract}

Palabras clave: papa amarilla / papa Tumbay / harina de papa / papa-producción

\section{Installation of a processing plant for mashed yellow potato with dehydrated additives}

\begin{abstract}
Peru has a remarkable variety of potatoes, but its low industrialization limits the development opportunities of producers. The project aims to develop the productive chain of the Tumbay yellow potatoe, in partnership with producers and gatherers. In order to achieve the project must generate a sustainable demand by satisfying the need of a nutritious, easy to prepare and palatable product for the Peruvian market and, in a longer term, for export.
\end{abstract}

Keywords: yellow potato / Tumbay potato / potato flour / potato-production 
A 4 mil metros sobre el nivel del mar viven Julio Hancco, su esposa Rosa y sus siete hijos. Tienen una casa humilde, una cocina humeante y unos escurridizos cuyes que chillan al comer. También tienen un tesoro, por el que Gastón Acurio considera a Julio (y a otros como él) 'general de la armada de nuestra biodiversidad': 186 tipos de papas nativas que crecen en sus parcelas del sector de Qanqaupata, en la comunidad de Pampacorral

(MUÑOZ 2010)

\section{INTRODUCCIÓN}

El Perú, que tras el estudio de las variedades nativas desarrolladas por los agricultores prehispánicos del norte del lago Titicaca, ha sido reconocido como el país originario de la papa, en la actualidad solo es responsable del $1,1 \%$ de la producción mundial del milenario cultivo, según información del Ministerio de Agricultura (Minag 2010).

En 1950, el consumo per cápita de papa en el país era de 120 kilogramos anuales, mucho mayor que los 13 kilogramos de consumo de arroz del momento. Cuatro décadas más tarde las cantidades habían cambiado: el peruano promedio consumía solo 30 kilogramos del tubérculo. Gracias a la importancia que ha retomado recientemente y las campañas de promoción realizadas, el consumo de la papa ha alcanzado un estimado de 90 kilogramos anuales (Muñoz 2010).

A pesar de ser el principal cultivo del país en superficie sembrada y de representar el $25 \%$ del PBI agropecuario, así como constituir la base de la alimentación de la zona andina y ser producido por 600 mil pequeñas unidades agrarias, su baja industrialización ha repercutido en el pobre desarrollo de la agricultura en la sierra central, lo que la convierte en actividad de supervivencia, de visión cortoplacista y por lo tanto de poca inversión y baja tecnificación.

Como resultado, el rendimiento por hectárea es el más bajo de Latinoamérica (Minag 2010) y aproximadamente la cuarta parte del de Estados Unidos (véase gráfico 1). 


\section{Gráfico 1}

Rendimiento promedio en t/ha en Latinoamérica

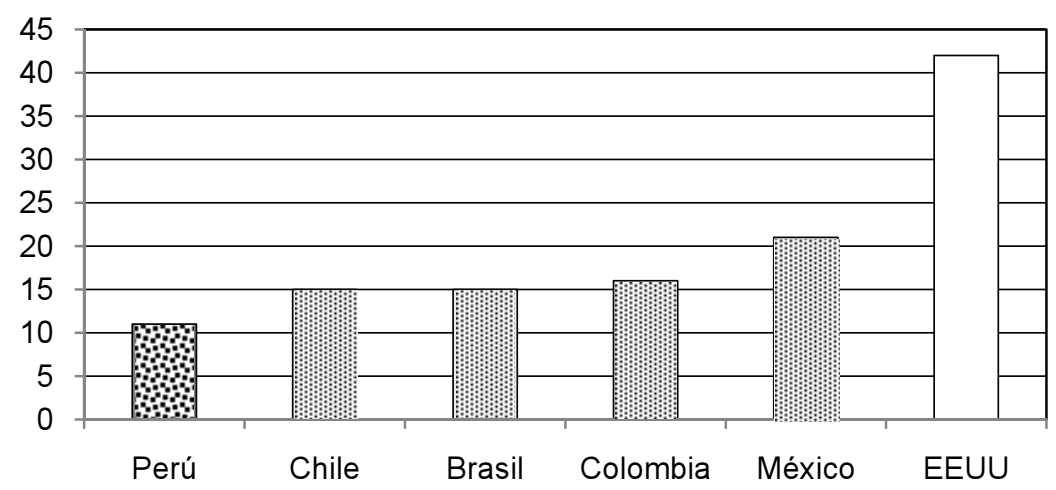

Fuente: Minag 2010.

El principal objetivo del proyecto es la creación de una empresa de viabilidad técnica y financiera sostenible, que trabaje en asociación con los productores y acopiadores de papa amarilla Tumbay, para generar una demanda sostenible al satisfacer la necesidad de la alimentación con un producto nutritivo, de fácil preparación y de buen sabor.

El desarrollo de la demanda para el producto endémico del Perú beneficiará la rentabilidad de la cadena productiva, lo que repercutirá en el nivel de vida del productor. El círculo virtuoso estará completo cuando, con apoyo de las organizaciones no gubernamentales (ONG) y por medio de asociaciones, los campesinos inviertan en la tecnificación del cultivo, medidas que harán que aumente el rendimiento, la calidad y la uniformidad del producto.

\section{DEFINICIÓN DEL PROYECTO}

Con el fin de generar la demanda inicial del proyecto, se producirá puré de papa instantáneo elaborado a partir de papa amarilla Tumbay, con distintos acompañamientos para darle nuevos sabores, como queso parmesano, espinaca, orégano, cebolla y ajo. 

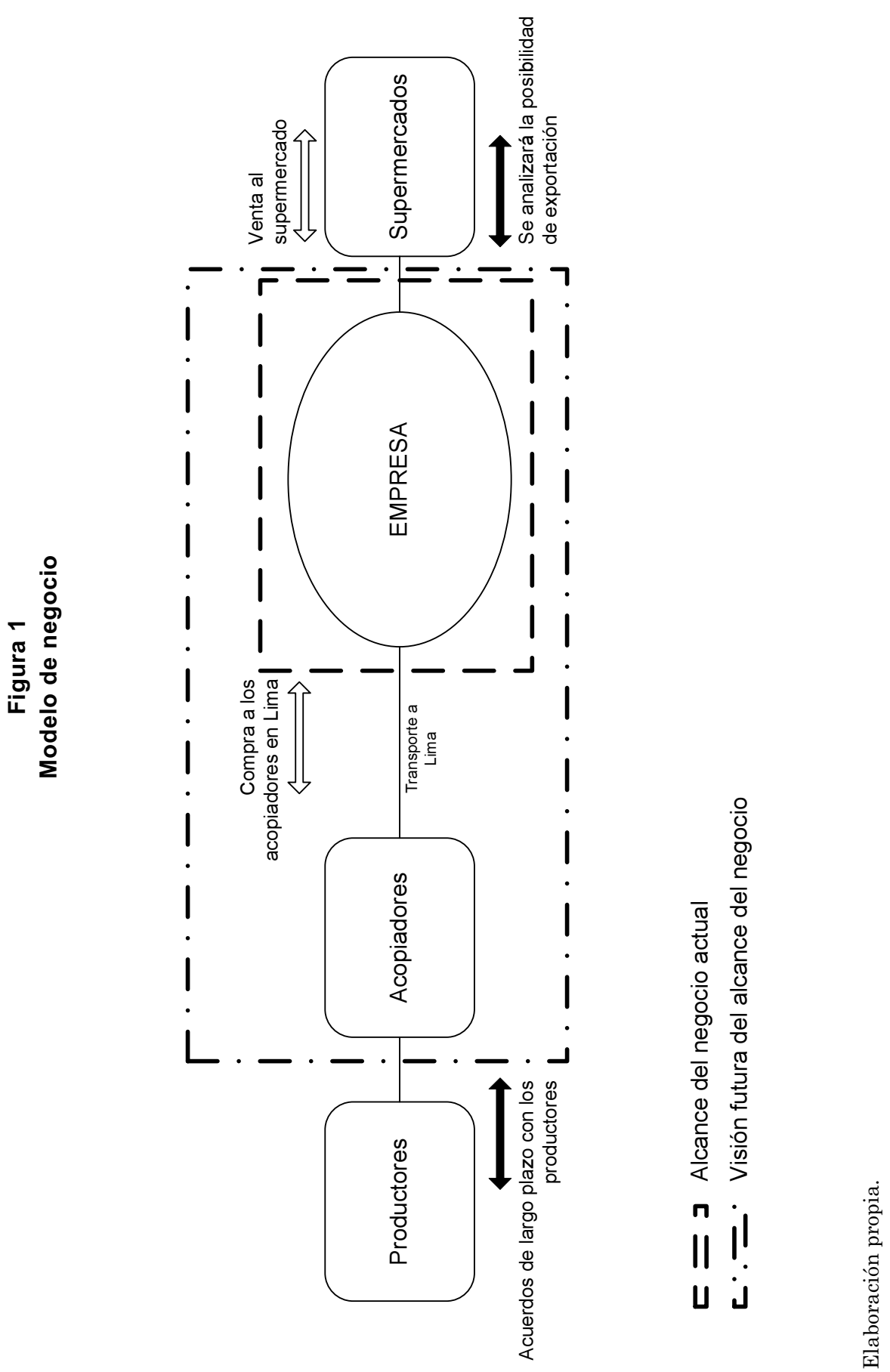
El producto estará dirigido principalmente a los niveles socioeconómicos (NSE) A, B y C. El NSE A es el que más consume puré en el país (29\% de la población de este NSE lo consume habitualmente); además, estaría dispuesto a pagar un poco más por nuevos y nutritivos sabores. Por otra parte, los sectores $\mathrm{B}$ y C podrán consumir menos en proporción, pero al estar constituidos por mayor número de habitantes es un mercado potencial para el proyecto.

El canal de abastecimiento para el proyecto se realizará mediante trato directo con los acopiadores y grupos de campesinos con acuerdos de volumen de compra asegurado a cambio de la producción bajo parámetros establecidos. Asimismo, el proyecto incluye en su presupuesto un monto destinado a mejorar y trabajar las relaciones con los acopiadores y posteriormente con los productores. Razón por la cual, en un plazo de 3 a 5 años se espera ampliar la cadena productiva de la empresa que, tras haber consolidado sus relaciones con los acopiadores, enfocará su presupuesto en el fortalecimiento de las relaciones con los productores (véase la figura 1).

La empresa invertirá en el crecimiento sostenible y la mutua confianza con sus proveedores con el objetivo de mejorar la eficiencia y calidad de la producción de papa amarilla Tumbay y otras papas nativas. El esfuerzo se realizará de manera conjunta con las ONG que buscan establecer acuerdos con los negocios que ofrezcan una demanda sostenible a los productores de la sierra central.

Por otra parte, se evaluará la posibilidad de ampliar el mercado de la empresa mediante la exportación de sus productos. Para este fin, deberán fortalecerse las relaciones con los productores con el fin de contar con el abastecimiento y la calidad de la materia prima requerida. Por ejemplo, para exportar productos orgánicos en la producción de la materia prima solo se utilizarán pesticidas e insecticidas adecuados, aspecto sobre el cual los productores deberán ser capacitados.

\section{ESTUDIO DE MERCADO}

\subsection{Demanda del proyecto}

La demanda del proyecto se ha estimado como una porción (participación de mercado) de la demanda de puré de papa de los segmentos socioeconómicos A y B en Lima metropolitana. 
Si se considera un crecimiento promedio histórico de $3,5 \%$ y una participación de mercado de $7,5 \%{ }^{1}$ en los próximos 7 años, se obtiene la siguiente demanda estimada:

\section{Cuadro 1}

Modelo de negocio

\begin{tabular}{cccc}
\hline Año & Ventas en t & $\begin{array}{c}\text { Ventas en miles } \\
\text { de bolsas de 125g }\end{array}$ & $\begin{array}{c}\text { Ventas en } \\
\text { miles de } \mathbf{S} / .\end{array}$ \\
\hline 2011 & 62,20 & 497,64 & $1.288,88$ \\
2012 & 64,63 & 517,07 & $1.339,20$ \\
2013 & 67,06 & 536,5 & $1.389,53$ \\
2014 & 69,49 & 555,93 & $1.439,85$ \\
2015 & 71,92 & 575,36 & $1.490,17$ \\
2016 & 74,35 & 594,79 & $1.540,50$ \\
2017 & 76,78 & 614,22 & $1.590,82$ \\
2018 & 79,21 & 633,65 & $1.641,15$ \\
\hline
\end{tabular}

Elaboración propia.

\subsection{Precio}

El precio del producto ha sido estimado principalmente sobre la base de dos factores: los precios de mercado y la encuesta de campo realizada.

Los precios de mercado oscilan entre $\mathrm{S} / .2,50$ y $\mathrm{S} / .2,85$ para la misma presentación, como se muestra en el cuadro 2. Por otra parte, según la encuesta realizada, el público objetivo estaría dispuesto a pagar un precio superior por ser un producto diferenciado con mejor sabor y nutrición.

1 Estimada en función de las 72 encuestas realizadas durante el estudio. 


\section{Cuadro 2}

Precios del puré de papa en el mercado nacional

\begin{tabular}{lc}
\multicolumn{2}{c}{ Precios en supermercados } \\
\hline Presentación & Precio (S/.) \\
\hline Puré de papa Menú x $125 \mathrm{~g}$. & 2,50 \\
Puré de papa Knorr x $125 \mathrm{~g}$. & 2,80 \\
Puré de papa Maggi x $125 \mathrm{~g}$. & 2,85 \\
\hline
\end{tabular}

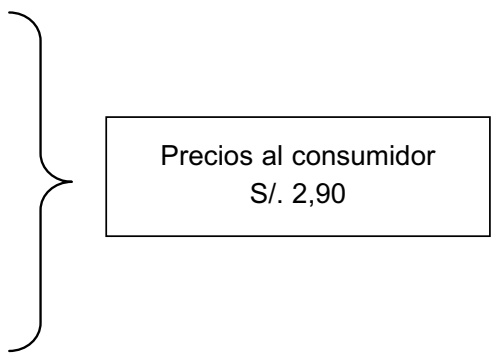

Elaboración propia.

\section{DISPONIBILIDAD DE INSUMOS}

La materia prima para el proyecto es la papa amarilla Tumbay.

Esta variedad ha sido reconocida alrededor del mundo por su sabor y valor nutritivo, muy superior al de la papa blanca convencional de la cual se hacen actualmente los purés de papa instantáneos. La papa Tumbay ha ganado premios en Francia y otros países de Europa, lo que ratifica su buena calidad y facilita la exportación del producto en estudio. Por otra parte, esta variedad solo se cultiva en el Perú, por lo que se tendría una ventaja competitiva nacional cuando se inicie la exportación del producto.

Es conocida como la mejor papa del mundo, pues sea cual fuere la forma de preparación su incomparable sabor es el resultado de su alto contenido de materia seca y aminoácidos; además, no tiene colesterol, lo que impide la acumulación de grasas (Proinversión). Adicionalmente, tiene la textura adecuada para la obtención de puré y otorga facilidad de cocción. 


\section{Cuadro 3 \\ Características morfológicas y agronómicas de la papa amarilla Tumbay}

\begin{tabular}{ll}
\hline & Características morfológicas y agronómicas \\
\hline Plantas & $\begin{array}{l}\text { Medianas, grandes y erectas, tallo verde con pigmentación } \\
\text { morada, hojas verde oscuras con interhojuelas. }\end{array}$ \\
\hline Flores & Color rojizo. Abundante floración y escasa fructificación. \\
\hline Tubérculos & $\begin{array}{l}\text { Redondos, en forma de piña, ojos profundos. Piel de color } \\
\text { amarillo claro. Carne amarilla, ojos profundos. }\end{array}$ \\
\hline Brotes & Color morado. \\
\hline Período vegetativo & Semitardío (150 días). \\
\hline Rendimiento & Hasta 20-30 t/ha. \\
\hline Adaptación & Sierra central: Huánuco, Pasco, Junín. \\
\hline Calidad culinaria & Excelente cocción y procesamiento. \\
\hline
\end{tabular}

Fuente: http://www.portalagrario.gob.pe/boletines/papa_variedades.pdf.

Elaboración propia.

A partir de un estudio de Maximixe (2004) sobre el mercado de la papa se analizaron distintas fortalezas y debilidades en cuanto a la producción y los productores de papa amarilla Tumbay.

\section{Cuadro 4 \\ Análisis de la producción de papa amarilla Tumbay}

\begin{tabular}{|c|c|}
\hline Fortalezas & Debilidades \\
\hline 1. Experiencia en el cultivo. & $\begin{array}{l}\text { 1. Gran parte de los agricultores tiene } \\
\text { recursos limitados. }\end{array}$ \\
\hline 2. Producción durante todo el año & 2. Bajo nivel de cultivo. \\
\hline $\begin{array}{l}\text { 3. Presenta ventajas de sabor, textura, } \\
\text { nutrición, facilidad en la cocción. }\end{array}$ & 3. Baja industrialización. \\
\hline 4. Producción en gran volumen & 4. Atomización de la producción \\
\hline
\end{tabular}

Elaboración propia. 
Se cosecha a más de 3.000 metros de altitud, en la sierra central del Perú, en los departamentos de Huánuco, Junín y Pasco.

El departamento de Huánuco es el principal productor de la variedad, debido a que tiene un clima uniforme durante todo el año, favoreciendo la producción y el abastecimiento constantes.

Actualmente, la producción de la materia prima asciende a 136.000 toneladas por año, de las cuales el proyecto solo requeriría 360 toneladas en el año de mayor demanda. Los precios de este insumo varían de S/.0,85 el kilo en Huánuco a S/.1,00 por kilo en Lima.

\section{INGENIERÍA DEL PROYECTO}

\subsection{Especificaciones técnicas del producto}

En el cuadro 5 se puede observar la ficha técnica del producto por ofrecer:

\section{Cuadro 5}

Ficha técnica del producto: Puré de papa en bolsa de $125 \mathrm{~g}$

\begin{tabular}{ll}
\hline Nombre común & Puré de papa amarilla Tumbay con queso parmesano \\
\hline Ingredientes & $\begin{array}{l}\text { Papa amarilla Tumbay deshidratada, queso parmesano } \\
\text { deshidratado }\end{array}$ \\
Presentación & $\begin{array}{l}1 \text { caja master = Display = 48 bolsas. Sabor deshidratado en } \\
\text { bolsa aparte. }\end{array}$ \\
Empaque & Bolsa trilaminado(PET/ALUMINIO7PEBD) sellada al vacío. \\
$\begin{array}{l}\text { Peso bolsa } \\
\text { Forma }\end{array}$ & $\begin{array}{l}125 \mathrm{~g} . \\
\text { Escamas de papa deshidratada. Malla 200. } \\
\text { Sabor }\end{array}$ \\
Humedad & Característico. \\
\hline
\end{tabular}

Fuente: A\&L Biodiversidad Altoandina.

Elaboración propia.

\subsection{Proceso productivo}

El proceso productivo se realiza de forma semiautomática en 18 actividades que incluyen operaciones y controles de calidad. Este se describe en el diagrama de operaciones de proceso. 


\section{Diagrama 3}

Diagrama de operaciones de proceso de elaboración de puré de papa amarilla Tumbay deshidratado con agregados
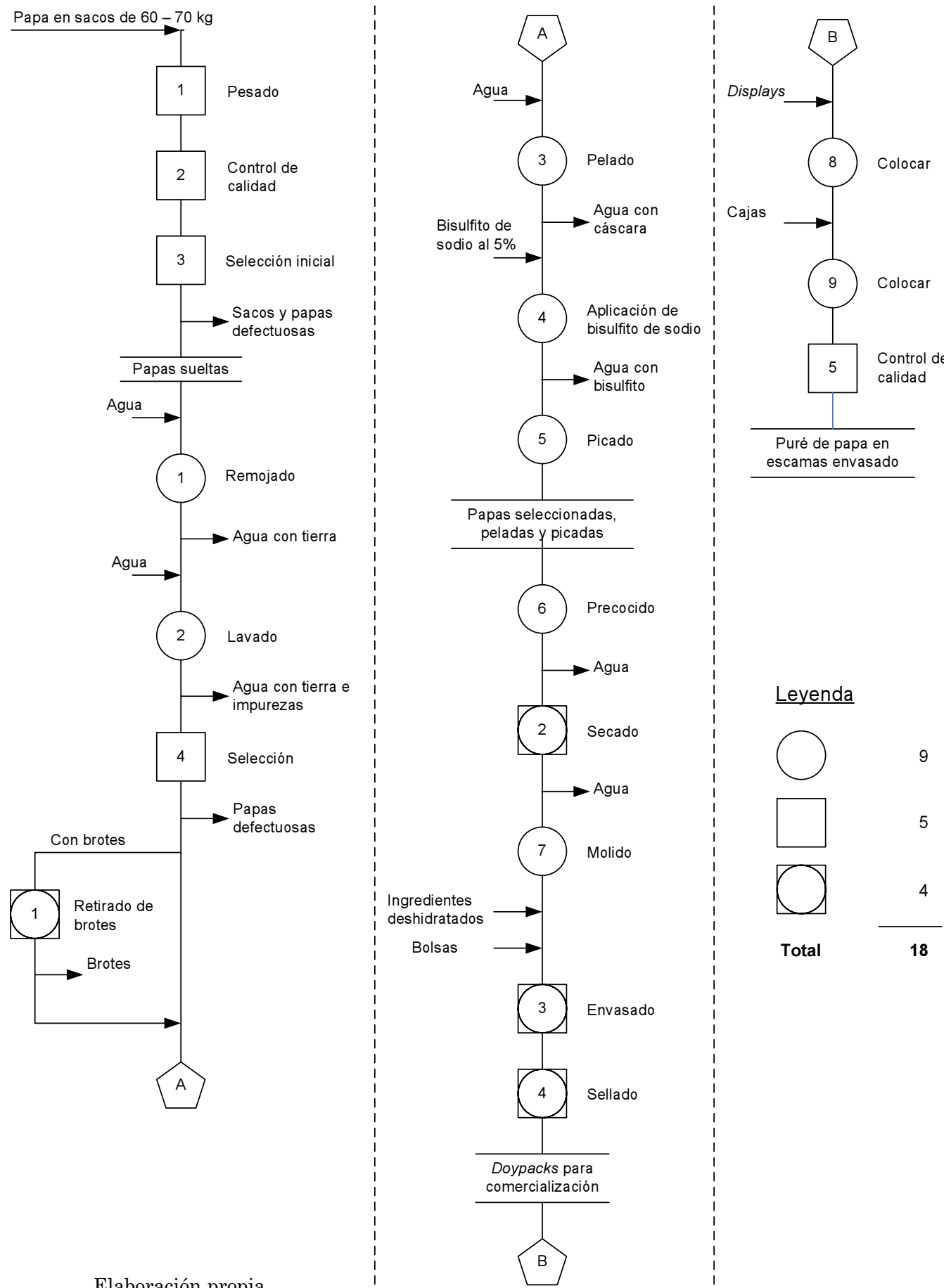

Elaboración propia. 


\subsection{Maquinaria, equipo y personal}

La relación de la maquinaria necesaria para la producción se resume a continuación:

\section{Cuadro 6}

Maquinaria requerida para el proceso

\begin{tabular}{lclc}
\hline Operación & $\begin{array}{c}\text { Núm. } \\
\text { máquinas }\end{array}$ & Descripción de las máquinas o proceso & $\begin{array}{c}\text { Capacidad } \\
\mathbf{k g} / \mathbf{~ m a q}\end{array}$ \\
\hline Pesado & 1 & Balanza de plataforma & 70 \\
Lavado & 1 & Lavadora de tubérculos & 70 \\
Pelado & 1 & Peladora & 10 \\
Picado & 1 & Picadora & 10 \\
Precocido & 3 & Cocina industrial & 60 \\
Secado & 1 & Secador de tambor & 180 \\
Molido & 1 & Molino de martillos & 60 \\
Sellado & 1 & Selladora & 1 \\
\hline
\end{tabular}

Elaboración propia.

La operación de mayor criticidad para la calidad del producto y cuello de botella es el proceso de secado. Este se realizará en un secador de tambor con motor de 3HP, donde se deberán controlar la temperatura, la humedad y la velocidad de rotación.

El personal requerido será de 14 personas: 3 de gestión (gerencia general, producción y logística) y 11 operarios.

\subsection{Gestión de calidad}

Debido a que se trata de un producto alimenticio, es de suma importancia controlar la calidad en la producción. Así, se usará el sistema HACCP (Hazard Analysis \& Critical Control Points), ejerciendo un constante análisis de riesgos y cuidando los puntos críticos de control identificados en el proceso. 


\section{ASPECTOS ECONÓMICOS}

\subsection{Inversión}

La inversión en el proyecto se detalla en el siguiente cuadro:

\section{Cuadro 7 \\ Inversión requerida para el proyecto}

\begin{tabular}{lc}
\hline Descripción & Monto total (US\$) \\
\hline Inversión fija intangible & $\mathbf{2 5 . 6 7 2 , 8 9}$ \\
Estudios previos & $4.814,81$ \\
Gastos de puesta en marcha & 740,74 \\
Ingeniería y supervisión & $5.397,33$ \\
Gastos durante la construcción & $6.378,66$ \\
Utilidad del contratista & $2.780,44$ \\
Contingencias & $5.560,89$ \\
Inversión fija tangible & $\mathbf{1 8 6 . 0 8 1 , 4 2}$ \\
Costo del terreno & $49.777,78$ \\
Gastos de infraestructura & $102.708,15$ \\
Máquinaria y equipos & $16.355,56$ \\
Muebles y enseres & $2.722,96$ \\
Instalaciones y montajes & $7.360,00$ \\
Imprevistos tangibles (4\% del tangible) & $7.156,98$ \\
\hline Subtotal & $\mathbf{2 1 1 . 7 5 4 , 3 1}$ \\
\hline Capital de trabajo (3 meses y medio) & $\mathbf{1 1 2 . 3 0 1 , 0 6}$ \\
Materia prima e insumos & $43.747,05$ \\
Energía eléctrica, agua y gas natural & $1.424,04$ \\
Mano de obra directa e indirecta & $49.784,00$ \\
Traslado y distribución & 215,30 \\
Gastos administrativos & $14.275,56$ \\
Gastos de relación con acopiadores & $2.855,11$ \\
\hline Subtotal & $\mathbf{1 1 2 . 3 0 1 , 0 6}$ \\
\hline Total & $\mathbf{3 2 4 . 0 5 5 , 3 7}$ \\
\hline
\end{tabular}

Elaboración propia. 
El 34,65\% de la inversión está destinada al capital de trabajo, debido a que se consideran 3,5 meses para poder cubrir el costo financiero de los pagos a 90 días que tienen como política algunos supermercados.

Otro aspecto que representa una fuerte inversión en el proyecto es la infraestructura, que en gran parte $(31,69 \%)$ se ven influenciadas por las instalaciones de gas natural en la planta, además de las inversiones en construcción (58\%).

\subsection{Evaluación económica}

Los ratios obtenidos tras la evaluación económica del proyecto se muestran en el siguiente cuadro:

\begin{tabular}{lc} 
Cuadro 8 \\
Indicadores económicos y financieros del proyecto \\
\hline VAN & $118.025,38$ \\
TIR & $26,85 \%$ \\
Relación B/C & 1.40 \\
Periodo de recupero & 5,67 \\
\hline
\end{tabular}

Elaboración propia.

Si bien el VAN obtenido es positivo y la TIR es mayor que el COK (16\%), ofreciendo una rentabilidad atractiva para un proyecto de riesgo moderado, la relación beneficio-costo no es del todo satisfactoria; más aún si se considera que los flujos no son muy voluminosos en el tiempo y que el periodo de recuperación es un plazo ligeramente mayor al promedio peruano. Sin embargo, el punto de equilibrio es altamente superado por la estimación de demanda anual, por lo que se esperaría un riesgo de mercado bajo y cierta certeza de que los flujos serán positivos en el tiempo.

\section{CONCLUSIONES}

- El proyecto en estudio es técnica y económicamente viable. Asimismo, el riesgo es moderado, aunque existe una alta dependencia de la materia prima. 
- Se generará un impacto positivo en la región de influencia debido a la generación de una demanda de largo plazo y capacitación para los productores.

- La inversión es relativamente baja y el potencial de desarrollo social bastante alto, por lo que se podría trabajar con una ONG.

- Existe un nicho en el mercado nacional para el producto, pero se recomienda hacer un análisis más profundo de la posibilidad de exportar el producto a Europa.

- El posicionamiento y las ventas del producto en estudio servirían de base para la creación de otros productos de mayor valor agregado, como las papas fritas en hojuelas, los gnocchis o la causa precocida, dado que se contaría con la harina de papa amarilla Tumbay.

\section{BIBLIOGRAFÍA}

Barbosa, Gustavo (2000). Deshidratación de alimentos. Zaragoza: Acribia.

Barrientos, Pedro (2000). "Marketing de los productos agrarios". Revista de la Facultad de Ciencias Económicas. Año V, núm. 17. Lima: Universidad Nacional Mayor de San Marcos.

Contreras, Catherine. "Sepa quiénes son los guardianes de nuestras papas nativas". El Comercio. <http://elcomercio.pe/ noticia 479500/ sepa-quienes-son-guardianes-nuestras-papas-nativas>. [Fecha de consulta: Junio del 2010.]

Dirección Regional Agraria Huánuco. "Portal agrario de Huánuco". $<$ http://www.huanucoagrario.gob.pe/>. [Fecha de consulta: Junio del 2010.]

Herrera, J. (Setiembre de 1994). "Perspectivas para el desarrollo agroindustrial de la papa en el Perú". Debate Agrario 19. Lima: Cepes.

Instituto Nacional de Estadística e Informática. "Índice de precios al consumidor". <http://www.inei.gob.pe/web/indices/default.htm>. [Fecha de consulta: Junio del 2010.]

Kalpakjian, Serope (2008). Manufactura, ingeniería y tecnología. México, D.F.: Prentice Hall.

Maximixe Consult (2004). Papa. Riesgos de mercados. Lima. 
Mayo, Raúl. "Las papas nativas conquistan el mundo". El Comercio. $<$ http://elcomercio.pe/noticia/395453/papas-nativas-conquistanmundo_1>. [Fecha de consulta: Junio 2010.]

Ministerio de Agricultura. "Estadísticas de producción, siembra y cultivos". <http://www.minag.gob.pe/herramientas/boletines.html>. [Fecha de consulta: Junio del 2010.]

Ministerio de Agricultura. "Información general sobre la papa". $<$ http://www.minag.gob.pe/cultivos-de-importancia-nacional/papa.html>. [Fecha de consulta: Junio del 2010.]

Muñoz Monge, Antonio. "La papa a través de las leyendas e historia" [en línea]. El Comercio. <http://elcomercio.pe/noticia/487457/papa-traves-leyendas-historia> [Fecha de consulta: Junio del 2010.]

Nave, Robert (Junio de 1996). "Procesando una sobreproducción de papa”. Cadena Alimentaria 1. 10-11. Lima.

Proinversión. Agencia de Promoción de la Inversión Privada. "Procesamiento de la papa amarilla". <http://www.proinversion. gob.pe/>. [Fecha de consulta: Junio del 2010.]

Shaw, Roy (1975). Simple processing of dehidrated potatoes and potato starch. Lima: CIP.

Superintendencia Nacional de Administración Tributaria. "Información de importaciones y exportaciones". <http://www.aduanet.gob.pe/>. [Fecha de consulta: Junio del 2010.] 